\title{
Optimal Laser Pulse Shaping for Interferometric Multiplex Coherent Anti-Stokes Raman Scattering Microscopy
}

\author{
Bi-Chang Chen and Sang-Hyun Lim* \\ Department of Chemistry and Biochemistry, University of Texas at Austin, 1 University Station A5300, \\ Austin, Texas, 78712
}

Received: November 9, 2007; In Final Form: December 19, 2007

\begin{abstract}
We present a significant sensitivity improvement of interferometric multiplex coherent anti-Stokes Raman scattering (CARS) by optimizing the power, bandwidth and phase of the pump, Stokes, and probe pulses independently. Fourier transform spectral interferometry (FTSI) is used to retrieve the entire complex quantity of the CARS spectrum by utilizing the non-resonant background as a local oscillator. Background-free spontaneous Raman-like vibrational spectra can be measured over the $500-1400 \mathrm{~cm}^{-1}$ range with $20 \mathrm{~cm}^{-1}$ spectral resolution within a tens of microseconds time scale. Chemically selective microscopy of a multicomponent polymer film is performed to demonstrate the feasibility of its microscopy application. A systematic analysis of the signal recovery method and several technical issues are discussed.
\end{abstract}

\section{Introduction}

Coherent anti-Stokes Raman scattering (CARS) is a thirdorder nonlinear vibrational spectroscopy technique, where the interaction of two different frequency laser pulses, $\omega_{\mathrm{P}}$ and $\omega_{\mathrm{S}}$ (pump and Stokes pulses) creates coherent vibrations with which a third pulse, $\omega_{\operatorname{Pr}}$ (probe pulse) generates anti-Stokes signals at the frequency $\omega_{\mathrm{AS}}=\omega_{\mathrm{P}}-\omega_{\mathrm{S}}+\omega_{\mathrm{Pr}}$ (Figure 1). ${ }^{1,2}$ Because of the coherent vibration excited by the pump and Stokes pulses, CARS can achieve much more sensitivity than the spontaneous Raman scattering spectroscopy. ${ }^{1}$ In addition, since CARS is a third-order nonlinear process, the signal is generated mostly at the tight focus of the overlapped incident laser beams, allowing three-dimensional depth profiling and a better lateral spatial resolution. ${ }^{3}$ It holds a great promise for label-free chemical imaging, and there has been much effort to increase the sensitivity and selectivity of the technique. ${ }^{4-15}$

CARS experiments typically combine two narrow bandwidth laser pulses (pulse duration of picoseconds to nanoseconds) with a frequency difference matching a single vibrational resonance of the sample molecule. ${ }^{16}$ The entire laser power is used to excite and detect a single vibrational resonance in this single-frequency CARS method. ${ }^{17}$ Recently, this technique has advanced greatly to achieve a sensitivity level of detecting $10^{4}-10^{5}$ oscillators in the time scale of microseconds ${ }^{7,18}$ and a video-rate laser scanning CARS microscopy has already been demonstrated. ${ }^{19,20}$ If a broadband laser pulse is used in the excitation laser pulse combination, CARS can be configured to measure a vibrational spectrum instead of a single vibrational peak. ${ }^{15,21}$ This multiplex CARS technique has also received much attention since it can detect a broadband vibrational spectrum in a single experimental measurement to provide great chemical selectivity by analyzing multiple vibrational peaks. ${ }^{8-10,12,13,22-25}$ The non-resonant signal, however, has been a major problem in this method. ${ }^{1}$ This unwanted background is generated by the electronic response of the sample and the use of a broadband laser pulse (a shorter pulse duration in the time domain) increases it significantly. ${ }^{1}$ It

* To whom correspondence should be addressed. E-mail: shlim@ mail.utexas.edu.

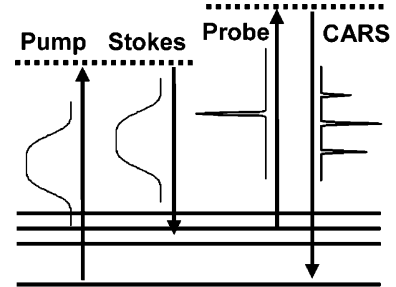

Figure 1. Multiplex CARS process. Note that the broadband pump and Stokes pulses create coherent vibrations and the probe pulse generates a CARS spectrum over the $500-1400 \mathrm{~cm}^{-1}$ range in the current experiments.

not only adds up as a background but also interferes with the weak resonant CARS signal to distort the spectral line shape, making the vibrational analysis difficult. ${ }^{26}$ The problem was solved when several groups applied interferometry to discriminate the resonant signal against the non-resonant background since the two signals have different phase responses. 5,6,10,11,13,22,27 Interferometry also increases the signal sensitivity significantly via the homodyne amplification with a strong local oscillator. ${ }^{5,22}$ However, the sensitivity of the multiplex CARS technique is still several orders of magnitude lower than that of the singlefrequency CARS since the laser power is distributed over many vibrations. The short laser pulse also induces more sample photodamage than the longer pulse, lowering the allowed laser power that a sample can tolerate. Nevertheless, its ability to detect a vibrational spectrum draws a great interest in the development of the technique. .,8-13,22,23,25 $^{-1}$

Fourier transform spectral interferometry CARS (FTSICARS) is one of interferometric multiplex CARS methods to extract the vibrationally resonant CARS spectrum against the non-resonant background. ${ }^{10}$ This method uses the inherent nonresonant background generated by the same laser pulse as a local oscillator for a spectral interferometry and retrieves a background-free Raman-like vibrational spectrum. It requires only a phase pulse shaping and vibrational spectrum over the entire detection window can be measured in a single measurement. ${ }^{10}$ Here we demonstrate that the signal sensitivity of the FTSI-CARS method can be improved further $(2$ orders of 
magnitude more than that found in ref 10) by an additional amplitude pulse shaping and use of a laser with a lower repetition rate. Vibrational spectra from liquid and polymer samples under a microscope are obtained in a time scale of tens of microseconds at a single spatial position. Chemical imaging of a polymer mixture sample is also performed to demonstrate the feasibility of the current technique in chemical imaging applications. Several technical issues such as broadband pulse compression at the sample position, axial chromatic aberration (ACA), the CARS spectral window, and the nonlinearity of sample photodamage are discussed.

This paper is organized as follows. In section II, we first consider the optimal power distribution, bandwidth, and phase shape of the three CARS excitation laser pulses (pump, Stokes, and probe pulses in Figure 1). Then in section III, we explain the FTSI-CARS method in detail to show how one can extract a Raman-like CARS spectrum from the measured signal. Experimental results with liquid and polymer samples are followed to show the current sensitivity in section V. Various technical issues are also discussed later.

\section{Optimal Amplitude and Phase Shape for Multiplex CARS Signal Generation}

We select the pump, Stokes, and probe pulses inside a single broadband laser pulse by a phase and amplitude pulse shaping technique. ${ }^{13,23}$ In this section, we consider the optimal amplitude and phase shape in the CARS excitation laser configuration. Our goal is to generate strong resonant CARS signals over the fingerprint region of organic molecules $\left(600-1800 \mathrm{~cm}^{-1}\right)$. Since the sample photodamage limits the maximum allowable laser power, we are seeking for the optimal laser pulse shape under a given total laser power.

It is easier to understand the CARS process by dividing it into the excitation and probing steps. ${ }^{23,28}$ The pump and Stokes pulses generate the coherent vibration (Raman coherence) in the excitation step. Then, the probe pulse scatters off the coherent vibration to generate the CARS signal (Figure 1).

Let us consider the excitation step first. $A(\Omega)$, the coherent vibrational excitation at the frequency of $\Omega$, is described by ${ }^{28}$

$$
\begin{aligned}
& A(\Omega)=\int_{0}^{\infty} \mathrm{d} \omega^{\prime} E_{\mathrm{P}}\left(\omega^{\prime}\right) E_{\mathrm{S}}^{*}\left(\omega^{\prime}-\Omega\right) \\
&=\int_{0}^{\infty} \mathrm{d} \omega^{\prime}\left|E_{\mathrm{P}}\left(\omega^{\prime}\right)\right|\left|E_{\mathrm{S}}^{*}\left(\omega^{\prime}-\Omega\right)\right| \exp \left(i \phi_{\mathrm{P}}\left(\omega^{\prime}\right)-\right. \\
&\left.i \phi_{\mathrm{S}}\left(\omega^{\prime}-\Omega\right)\right)
\end{aligned}
$$

where $E_{\mathrm{P}}$ and $E_{\mathrm{S}}$ are the electric fields of pump and Stokes laser pulses, respectively. $\phi_{\mathrm{P}}(\omega)$ and $\phi_{\mathrm{S}}(\omega)$ are the phase of pump and Stokes pulses at the frequency of $\omega$, respectively. As one can see in eq $1, A(\Omega)$ is maximized when $E_{\mathrm{P}}$ and $E_{\mathrm{S}}$ are transform limited (i.e., $\phi_{\mathrm{P}}\left(\omega^{\prime}\right)-\phi_{\mathrm{S}}\left(\omega^{\prime}-\Omega\right)=0$ ). There is, however, a choice of bandwidth for $E_{\mathrm{P}}$ and $E_{\mathrm{S}}$ in the multiplex CARS experiment. In Figure 2a, we compare two different amplitude shapes of pump/Stokes pulse combinations with the same total laser power. Since the Stokes pulse has a broad bandwidth, one can achieve a reasonable vibrational window in both cases. $A(\Omega)$ over $0-600 \mathrm{~cm}^{-1}$ is excited by either the two pump or two Stokes pulses. The $600-1800 \mathrm{~cm}^{-1}$ region is from the pulse combination of the pump and Stokes pulses. Here we are only interested in optimizing the excitation over 600$1800 \mathrm{~cm}^{-1}$, which corresponds to the vibrational fingerprint region of organic molecules. Note that the pump pulse with a narrow bandwidth (Pump 1 in Figure 2a) is used in typical
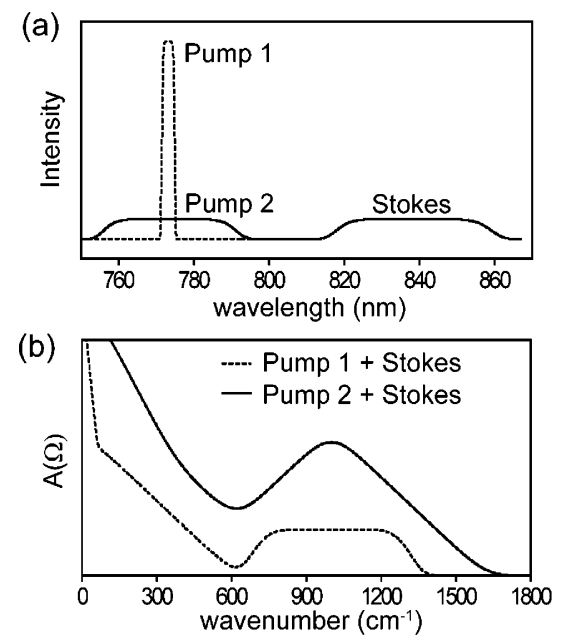

Figure 2. (a) Spectra of two different pump/Stokes pulse shapes used in (b) the simulation of coherent vibration excitations, $A(\Omega)$. The powers of pump 1 (dotted line) and 2 (solid line) are set to be equal. The Stokes pulse is identical for both simulations.

multiplex CARS experiments. ${ }^{8,15}$ Figure $2 \mathrm{~b}$ shows the simulated $A(\Omega)$ with the two pump/Stokes pulse configurations in Figure $2 \mathrm{a}$ and eq 1 . Stronger and broader $A(\Omega)$ is generated by the broadband pump pulse (solid line in Figure $2 \mathrm{~b}$ ). This can be understood by the following consideration. Since $A(\Omega)$ is essentially a convolution of the pump and Stokes electric fields, the amplitude of $A(\Omega)$ depends linearly on both the amplitude and bandwidth of $E_{\mathrm{P}}(\omega)$, i.e., $A \propto \int E_{\mathrm{P}}(\omega) \mathrm{d} \omega$. The pump power $\left(I_{\mathrm{P}}\right)$, however, has a different dependence on the amplitude of the electric field, $I_{\mathrm{P}}=\int\left|E_{\mathrm{p}}(\omega)\right|^{2} \mathrm{~d} \omega$. This leads to a larger amplitude of $A(\Omega)$ with a broadband pump pulse. There is, however, a complicating experimental factor. The sample photodamage is most likely nonlinear with femtosecond laser pulses, and a shorter pump pulse (broader bandwidth) causes more photodamage than a longer pulse with the same laser power. ${ }^{20,29}$ Accordingly, these two factors should be compromised to generate the optimal signal. In experiments with polymer samples, we find that the pump pulse with $\sim 300 \mathrm{~cm}^{-1}$ bandwidth generates the optimum CARS signal.

The next step is to scatter off the generated coherent vibration by the probe pulse. Since we wish to generate a vibrational spectrum with a high spectral resolution, the probe pulse should have a narrow bandwidth. ${ }^{12,13,23}$ Note that we are using separate pump and probe pulses, ${ }^{12,13,23}$ which is different from typical multiplex CARS experiments where a degenerate pump and probe pulse is used. ${ }^{8,9,21}$ In the current experiment, we select the probe pulse primarily by the phase pulse shaping since the pump and probe pulses are taken in the same frequency region of the laser. Since the resonant CARS signal generated by this narrow phase-shifted probe pulse has a different phase than the non-resonant signal, we can extract the resonant signal with the help of FTSI. ${ }^{10}$ The extraction method is explained in section III. Here we consider the optimal probe phase shape to achieve a high spectral resolution with a given probe bandwidth. Let us consider the two different phase masks for the probe pulse (Figure $3 \mathrm{a}$ and $\mathrm{b}$ ). Note that the two probe pulses have the same bandwidth. Figure 3 a shows a probe pulse with a flat spectral phase (flat phase probe), and this is what the previous demonstration used. ${ }^{10}$ The flat phase probe, however, also generates coherent vibrational excitation acting as a pump pulse. This is not a serious problem as long as the power of the narrow band probe pulse is small compared to that of the broadband 


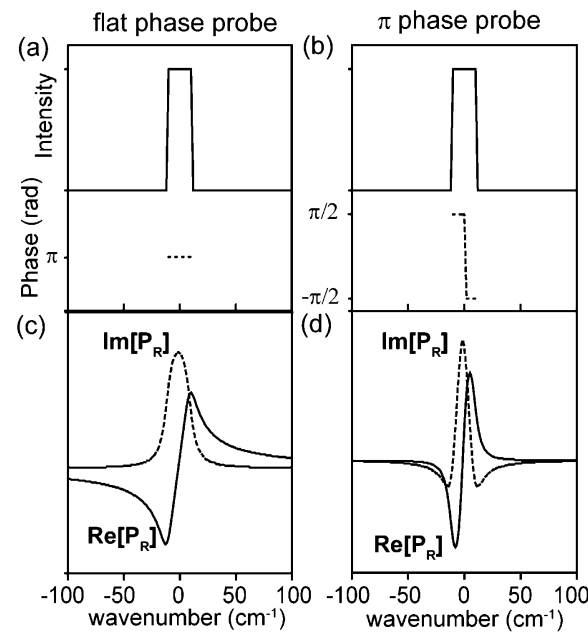

Figure 3. Amplitude and phase shape of the (a) flat phase and (b) $\pi$ phase probes. Also shown are the real and imaginary parts of the simulated resonant CARS signals $\left(P_{\mathrm{R}}\right)$ from a single vibrational resonance $\left(\Gamma=5 \mathrm{~cm}^{-1}\right)$ by (c) the flat phase probe and (d) the $\pi$ phase probe, respectively. Note that the bandwidths of the both probes are identical $\left(20 \mathrm{~cm}^{-1}\right)$.

pump pulse. If the probe pulse has a significant intensity, the excitation $(A(\Omega))$ begins to have a non-negligible component generated by the phase-shifted probe pulse, and the phase structure of $A(\Omega)$ becomes complicated. This can be avoided by using a $\pi$ step phase pulse ( $\pi$ phase probe), shown in Figure 3b. ${ }^{12,30}$ Let us consider $A(\Omega)$ excited by the $\pi$ phase probe as a pump pulse. Since the Stokes pulse $E_{\mathrm{S}}(\omega)$ has a much broader bandwidth than the probe pulse, eq 1 becomes

$$
\begin{aligned}
A(\Omega) & =\int_{0}^{\infty} \mathrm{d} \omega^{\prime} E_{\mathrm{Pr}}\left(\omega^{\prime}\right) E_{\mathrm{S}}^{*}\left(\omega^{\prime}-\Omega\right) \\
& =\int_{\omega_{\mathrm{Pr}}-\Delta}^{\omega_{\mathrm{Pr}}} \mathrm{d} \omega^{\prime} E_{\mathrm{Pr}}\left(\omega^{\prime}\right) E_{\mathrm{S}}^{*}\left(\omega^{\prime}-\Omega\right)+ \\
& \int_{\omega_{\mathrm{Pr}}}^{\omega_{\mathrm{Pr}}+\Delta} \mathrm{d} \omega^{\prime} E_{\mathrm{Pr}}\left(\omega^{\prime}\right) E_{\mathrm{S}}^{*}\left(\omega^{\prime}-\Omega\right) \\
& \approx\left|E_{\mathrm{Pr}}\left(\omega_{\mathrm{Pr}}\right)\right|\left(\mathrm{e}^{i \pi / 2}+\mathrm{e}^{-i \pi / 2}\right) E_{\mathrm{S}}^{*}\left(\omega_{\mathrm{Pr}}-\Omega\right) \Delta
\end{aligned}
$$

where $E_{\operatorname{Pr}}$ is the electric field of the probe, and $\omega_{\operatorname{Pr}}$ is the center frequency of the probe pulse. $\Delta$ is the half bandwidth of the probe (our probe pulse has an amplitude shape of a boxcar). Under this condition, the integration in eq 2 cancels, i.e.,

$$
A(\Omega) \approx\left|E_{\mathrm{Pr}}\left(\omega_{\mathrm{Pr}}\right)\right|(i-i) E_{\mathrm{S}}^{*}\left(\omega_{\mathrm{Pr}}-\Omega\right) \Delta=0
$$

So the $\pi$ probe pulse does not excite coherent vibrations with a broadband Stokes pulse. This is an important aspect since one can use a high-intensity narrow band probe pulse without generating a complicated phase structure in the resulting CARS spectrum.

The resonant and non-resonant CARS signals are described by $^{28}$

$$
\begin{gathered}
P_{\mathrm{R}}(\omega)=\int_{0}^{\infty} \mathrm{d} \Omega \chi_{\mathrm{R}}(\Omega) E_{\mathrm{Pr}}(\omega-\Omega) A(\Omega) \\
P_{\mathrm{NR}}(\omega)=\int_{0}^{\infty} \mathrm{d} \Omega \chi_{\mathrm{NR}}(\Omega) E_{\mathrm{Pr}}(\omega-\Omega) A(\Omega)
\end{gathered}
$$

where $P_{\mathrm{R}}$ and $P_{\mathrm{NR}}$ represent the polarizations of the resonant and non-resonant CARS signals, respectively. $\chi_{\mathrm{R}}$ and $\chi_{\mathrm{NR}}$ are the third-order nonlinear susceptibilities for the resonant and non-resonant CARS processes, respectively. The vibrational susceptibility $\chi_{\mathrm{R}}$ has the Lorentzian spectral line shape, i.e.,

$$
\chi_{\mathrm{R}}(\Omega) \equiv \alpha_{\mathrm{R}} \sum_{k} \frac{a_{k}}{\left(\Omega_{k}-\Omega\right)+i \Gamma_{k}}
$$

where $a_{k}, \Omega_{k}$, and $\Gamma_{k}$ are the relative intensity, frequency, and line width of vibrational mode $k$, respectively. Note that $\chi_{\mathrm{R}}$ has a $\pi$ phase shift at every vibrational resonance. ${ }^{23}$ The nonresonant susceptibility is usually approximated by ${ }^{28}$

$$
\chi_{\mathrm{NR}}(\Omega) \equiv \alpha_{\mathrm{NR}} / \Omega
$$

In eqs 5 and $6, \alpha_{R}$ and $\alpha_{N R}$ represent the amplitude coefficients of the resonant and non-resonant susceptibilities, respectively, responsible for the wavelength-independent factors such as concentration, electronic polarizability, and so forth. ${ }^{23}$ Note that $\alpha_{N R} / \alpha_{R}$ determines the relative intensity ratio between the non-resonant and resonant CARS signals. ${ }^{23}$

The $\pi$ probe pulse compensates the $\pi$ phase shift of $\chi_{\mathrm{R}}$ to maximize the CARS signal generation and also yields a narrow vibrational line width. ${ }^{30}$ As pointed out by Silberberg and coworkers, the probe phase, $\phi(\omega)=\arctan \left(\left(\omega-\omega_{\mathrm{Pr}}\right) / \Gamma\right)$ is optimal for the resonant CARS signal generation. ${ }^{30}$ The closest phase shape that we can produce with our pulse shaper is the one shown in Figure 3b due to the limited spectral resolution of the liquid crystal spatial light modulator. Figure $3 \mathrm{c}, \mathrm{d}$ shows the real and imaginary parts of the simulated CARS signals by the flat phase and $\pi$ phase probes, respectively. In the simulation, we set the probe bandwidth to $20 \mathrm{~cm}^{-1}$ and use a single vibrational resonance with $\Gamma=5 \mathrm{~cm}^{-1}$ in eq 5 . One can see that the vibrational line width with the $\pi$ probe pulse is about twice narrower than that by the flat phase probe (Figure $3 \mathrm{c}, \mathrm{d}$ ). ${ }^{30}$ So the $\pi$ phase probe is a good choice in terms of the spectral resolution too. There is, however, a problem with the line shape of the CARS spectrum generated by the $\pi$ phase probe. In Figure $3 \mathrm{~d}$, neither the real nor imaginary part of the CARS signal has the Raman-like line shape. This is due to the $\pi$ step phase structure of the probe pulse involved in eq 3 . We find that a Raman-like line shape can be obtained if the entire complex quantity of $P_{\mathrm{R}}(\omega)$ is available. The sum of the amplitude and imaginary part of $P_{\mathrm{R}}(\omega)$ shows a high spectral resolution with a Raman-like line shape, as shown in Figure 4a. In Figure 4, the CARS simulation is performed with the 1001 and $1028 \mathrm{~cm}^{-1}$ vibrational peaks from toluene $\left(\Gamma=5 \mathrm{~cm}^{-1}\right.$, and the probe bandwidth is $20 \mathrm{~cm}^{-1}$ ). One can see that the $\pi$ phase probe achieves a better spectral resolution than the flat phase probe, as shown in Figure 4b. Note that the simulated CARS spectra in Figure $4 \mathrm{~b}$ show the sum of the amplitude and the imaginary part for the signal by the $\pi$ phase probe and only the imaginary part for that by the flat phase probe.

\section{Fourier Transform Spectral Interferometric Coherent Anti-Stokes Raman Scattering (FTSI-CARS)}

In the previous section, we show that the $\pi$ phase probe does not excite coherent vibrations and is efficient to generate a CARS spectrum with a narrow vibrational line shape. The broadband pump pulse (pump 2 in Figure 2a), however, does not have this discriminating property. It also generates a CARS signal by acting as a probe pulse to yield orders of magnitude larger spectrally broad signals due to the short pulse duration. In other words, the spectrally resolved resonant CARS signal accompanies a much larger background. ${ }^{13,22}$ This background signal has two components from different origins. One is the non-resonant CARS signal, and the other is broad vibrational resonant CARS signals probed by the pump part of the laser pulse. ${ }^{23}$ Note that both backgrounds have very smooth spectral 
(a)

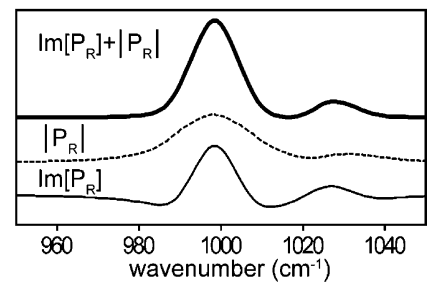

(b)

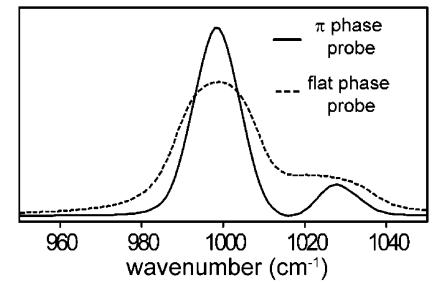

Figure 4. (a) Simulated imaginary part $\left(\operatorname{Im}\left[P_{\mathrm{R}}\right]\right)$, amplitude $\left(\left|P_{\mathrm{R}}\right|\right)$ and a sum of the imaginary part and amplitude $\left(\operatorname{Im}\left[P_{\mathrm{R}}\right]+\left|P_{\mathrm{R}}\right|\right)$ of the resonant CARS signal by the $\pi$ phase probe. Spectral parameters from Raman spectrum of toluene is used in the simulation (resonance at 1001 and $1028 \mathrm{~cm}^{-1}, \Gamma=5 \mathrm{~cm}^{-1}$ ). Each trace is displaced vertically for clarity. (b) Simulated toluene CARS signals: $\operatorname{Im}\left[P_{\mathrm{R}}\right]+\left|P_{\mathrm{R}}\right|$ by the $\pi$ phase probe (solid line) and $\operatorname{Im}\left[P_{\mathrm{R}}\right]$ by the flat phase probe (dotted line). Note that the bandwidths of the two probes are identical $\left(20 \mathrm{~cm}^{-1}\right)$.
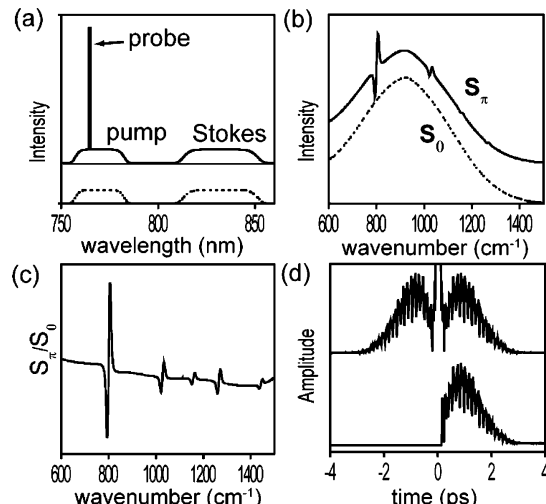

(e)

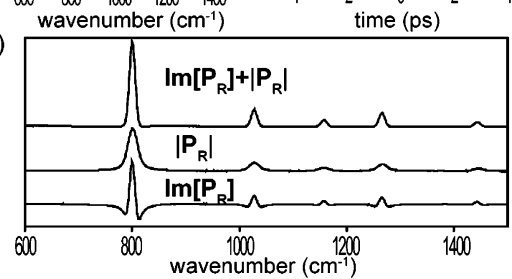

Figure 5. FTSI-CARS simulations: (a) The CARS (top) and the reference (bottom) laser pulse shapes used in the simulation. The CARS laser pulse has the $\pi$ phase probe at the probe frequency region, and the reference laser pulse is transform-limited. (b) Simulation of cyclohexane CARS signals by the CARS laser pulse $\left(\mathrm{S}_{\pi}\right.$, solid line) and the reference laser pulse ( $S_{0}$, dotted line). (c) Normalized CARS signal $\left(S_{\pi} / S_{0}\right)$, which corresponds to the cross term in eq 7. (d) (top) FT of the normalized signal $\left(S_{\pi} / S_{0}\right)$. (bottom) Zero-filled (before the time zero) time profile to recover the entire complex quantity of $P_{R}$. (e) imaginary part (bottom), amplitude (middle), and a sum of the imaginary part and amplitude (top) of the inverse FT of the bottom trace in (d). Traces in panels $b, d$, and e are vertically displaced for clarity.

amplitude and phase shapes due to the broad bandwidth of the pump pulse. In most condensed samples, we find that the nonresonant component is much larger than the broad resonant one. ${ }^{23}$ This means that the background has a smooth spectral shape and is almost transform-limited. Next we explain how we can use the background as a local oscillator to extract a narrow vibrational CARS spectrum.

Figure 5a shows the two CARS excitation laser spectra used in the simulation. The top trace in Figure 5a is the CARS excitation laser pulse with a distinct high-intensity probe pulse with the $\pi$ step phase (top trace). The $\pi$ step phase of Figure $3 \mathrm{~b}$ is applied in the narrow probe region. We also simulate the CARS spectrum with the reference excitation laser pulse (bottom trace in Figure 5a), which has a flat phase spectrum (i.e., transform limited) over the entire laser bandwidth. Figure $5 \mathrm{~b}$ shows the simulated CARS signals $\left(S_{\pi}\right.$ and $\left.S_{0}\right)$ from cyclohexane (resonances at $801,1028,1158,1266$, and $1444 \mathrm{~cm}^{-1}$ ) 31 by the CARS and reference excitation laser pulses, respectively. We adjust the $\alpha_{N R} / \alpha_{R}$ to match the relative amplitude of the interference fringe at $800 \mathrm{~cm}^{-1}$ to the experimental result (Figure $8 \mathrm{a})$. Note that the spectral shape of the reference signal $\left(S_{0}\right)$ has very little dependence on the vibrational response of the sample since the non-resonant signal dominates in this signal.

One can see that the narrow spectrally resolved CARS signals appear on top of the broad background in the top trace of Figure $5 b\left(S_{\pi}\right)$. This is in fact the cross term in the spectral interference between the non-resonant $\left(P_{\mathrm{NR}}(\omega)\right)$ and spectrally resolved resonant $\left(P_{\mathrm{R}}(\omega)\right)$ signals. ${ }^{10,13,23}$ Under the approximation of $P_{\mathrm{NR}}(\omega) \gg P_{R}(\omega)$, the measured signal, $S_{\pi}(\omega)$ becomes

$$
\begin{array}{r}
S_{\pi}(\omega) \propto\left|P_{\mathrm{NR}}(\omega)+P_{\mathrm{R}}(\omega)\right|^{2} \approx P_{\mathrm{NR}}(\omega)^{2}+P_{\mathrm{NR}}(\omega)\left(P_{\mathrm{R}}(\omega)+\right. \\
\left.P_{\mathrm{R}}(\omega)^{*}\right)(7)
\end{array}
$$

where $P_{\mathrm{NR}}(\omega)$ is the non-resonant signal by the CARS excitation laser pulse. One can get the cross term $\left(P_{\mathrm{R}}(\omega)+P_{\mathrm{R}}(\omega)^{*}\right)$ by normalizing the CARS signal $\left(S_{\pi}(\omega)\right)$ with the reference signal $\left(S_{0}\right)$. The normalized signal becomes

$$
S_{\pi}(\omega) / S_{0}(\omega) \approx\left|\frac{P_{\mathrm{NR}}(\omega)}{P_{\mathrm{NR}}^{0}(\omega)}\right|^{2}+\frac{P_{\mathrm{NR}}(\omega)}{\left|P_{\mathrm{NR}}^{0}(\omega)\right|^{2}}\left(P_{\mathrm{R}}(\omega)+P_{\mathrm{R}}(\omega)^{*}\right)
$$

where $P_{\mathrm{NR}}^{0}(\omega)$ is the non-resonant signal by the reference excitation laser pulse. Figure $5 \mathrm{c}$ shows the normalized CARS signal $\left(S_{\pi} / S_{0}\right)$ from Figure $5 \mathrm{~b}$. Since the spectral shape of the non-resonant signal does not depend on the sample and the $\pi$ probe part of the laser pulse does not excite the non-resonant signals, the first term in eq $8\left(\left|P_{\mathrm{NR}}(\omega) / P_{\mathrm{NR}}^{0}(\omega)\right|^{2}\right)$ becomes a constant offset. The non-resonant contribution in the second term $\left(P_{\mathrm{NR}}(\omega) /\left|P_{\mathrm{NR}}^{0}(\omega)\right|^{2}\right)$ also has a very smooth frequency dependence compared to the resonant signal $\left(P_{\mathrm{R}}(\omega)+P_{\mathrm{R}}(\omega)^{*}\right)$. As a result, $S_{\pi} / S_{0}$ has the spectral line shape of $P_{\mathrm{R}}(\omega)+P_{\mathrm{R}}(\omega)^{*}$, which is the real part of the resonant CARS signal (Figure $5 \mathrm{c}$ ). We can retrieve the complex function of $P_{\mathrm{R}}(\omega)$ out of $P_{\mathrm{R}}(\omega)+$ $P_{\mathrm{R}}(\omega)^{*}$ by the following method.

So far we consider CARS signals only in the frequency domain. In the time domain, it has an interesting symmetry property, which we use to extract the full complex quantity of $P_{\mathrm{R}}(\omega)$ out of $P_{\mathrm{R}}(\omega)+P_{\mathrm{R}}(\omega)^{*}$. Let us consider Fourier transformation (FT) of Figure 5c. The upper trace in Figure 5d shows the FT of Figure 5c (the normalized CARS signal). This is essentially the FT of the real part of a Lorentzian function convoluted with the $\pi$ phase probe (eq 3 ). It is a well-known fact that an FT of a Lorentzian function has zero amplitudes before the time zero and has an exponential decay of the amplitude after the time zero. ${ }^{32,33}$ Mathematically,

$$
\begin{aligned}
& \mathrm{FT}\left(\frac{1}{\left(\Omega_{0}-\Omega\right)+i \Gamma_{0}}\right) \\
& \quad=\frac{1}{\sqrt{2 \pi}} \int_{-\infty}^{\infty} \mathrm{d} \omega \mathrm{e}^{i \omega t} \frac{1}{\left(\Omega_{0}-\Omega\right)+i \Gamma_{0}} \\
& \quad=0 \text { for } t<0 \\
& \quad=\sqrt{2 \pi} \exp \left(-\Gamma_{0} t\right) \exp \left(i \Omega_{0} t-\pi i / 2\right) \text { for } t>0
\end{aligned}
$$


It is also interesting to compare the FTs of $P_{\mathrm{R}}(\omega)$ and $P_{\mathrm{R}}(\omega)^{*}$. An FT of a complex conjugate quantity in the frequency domain is equivalent to an inversion in the time domain. ${ }^{32,33}$ This can be easily proved by

$$
\begin{aligned}
\mathrm{FT}\left[P_{\mathrm{R}}(\omega)^{*}\right]= & \frac{1}{\sqrt{2 \pi}} \int_{-\infty}^{\infty} \mathrm{d} \omega \mathrm{e}^{i \omega t} P_{\mathrm{R}}(\omega)^{*}= \\
& {\left[\frac{1}{\sqrt{2 \pi}} \int_{-\infty}^{\infty} \mathrm{d} \omega \mathrm{e}^{-i \omega t} P_{\mathrm{R}}(\omega)\right] *=P_{\mathrm{R}}(-t)^{*} }
\end{aligned}
$$

In other words, $P_{\mathrm{R}}(\omega)$ and $P_{\mathrm{R}}(\omega)^{*}$ are separated in the time domain. ${ }^{33}$ Note that a convolution with the $\pi$ phase probe changes the result of eq 9 , but $P_{\mathrm{R}}(\omega)$ and $P_{\mathrm{R}}(\omega)^{*}$ are still separated in the time domain. If we apply the Heaviside step function, $\theta(t)$, to the FT of the normalized CARS signal of eq 8 (the lower trace in Figure $5 \mathrm{~d}$ ), we can select only $P_{\mathrm{R}}(t)$, i.e.,

$$
\theta(t) \mathrm{FT}\left[P_{\mathrm{R}}(\omega)+P_{\mathrm{R}}(\omega)^{*}\right]=\mathrm{FT}\left[P_{\mathrm{R}}(\omega)\right]
$$

Then an inverse FT recovers the full complex quantity of $P_{\mathrm{R}}(\omega) .^{10}$

$$
\mathrm{FT}^{-1}\left[\theta(t) \mathrm{FT}\left[P_{\mathrm{R}}(\omega)+P_{\mathrm{R}}(\omega)^{*}\right]\right]=P_{\mathrm{R}}(\omega)
$$

Figure 5e shows the imaginary (bottom), amplitude (middle), and the sum of imaginary and amplitude parts (top) of the retrieved $P_{\mathrm{R}}(\omega)$. As we discuss in section II, one can see that $\operatorname{Im}\left[P_{\mathrm{R}}\right]+\left|P_{\mathrm{R}}\right|$ has the Raman-like line shape without any background.

It is worth considering the relationship of the current FTSICARS method with the Kramers-Kronig relations. As one may realize, what we are doing is transforming the real part of a CARS signal to the imaginary part of it. In fact, this is one of the Kramers-Kronig relations, ${ }^{2}$

$$
\operatorname{Im}[\chi(\omega)]=\frac{1}{\pi} \int_{0}^{\infty} \mathrm{d} \omega_{0} \frac{\operatorname{Re}\left[\chi\left(\omega_{0}\right)\right]}{\omega_{0}-\omega}
$$

where $\chi(\omega)$ is a general susceptibility. Our FTSI-CARS method can be reformulated with the convolution theorem ${ }^{32}$ (i.e., a multiplication in the time domain is equivalent to a convolution in the frequency domain). Equation 11 can be rewritten as

$$
\begin{gathered}
\mathrm{FT}^{-1}\left[\theta(t) \mathrm{FT}\left[P_{\mathrm{R}}(\omega)+P_{\mathrm{R}}(\omega)^{*}\right]\right] \\
=\mathrm{FT}^{-1}\left[\theta(t) \mathrm{FT}\left[2 \mathrm{Re}\left[P_{\mathrm{R}}(\omega)\right]\right]\right] \\
=\frac{1}{\sqrt{2 \pi}} \mathrm{FT}^{-1}[\theta(t)] \otimes \mathrm{FT}^{-1}\left[\mathrm{FT}\left[2 \operatorname{Re}\left[P_{\mathrm{R}}(\omega)\right]\right]\right] \\
=\frac{1}{\sqrt{2 \pi}} \mathrm{FT}^{-1}[\theta(t)] \otimes 2 \operatorname{Re}\left[P_{\mathrm{R}}(\omega)\right]
\end{gathered}
$$

where $\otimes$ is a convolution operator. With $\mathrm{FT}^{-1}[\theta(t)]=1 /(i$ $\sqrt{2 \pi} \omega)+\sqrt{\pi / 2} \delta(t),{ }^{32}$ eq 13 becomes

$$
\begin{aligned}
\mathrm{FT}^{-1}\left[\theta(t) \mathrm{FT}\left[P_{\mathrm{R}}(\omega)+P_{\mathrm{R}}(\omega)^{*}\right]\right] \\
=\frac{1}{\sqrt{2 \pi}}\left(\frac{1}{i \sqrt{2 \pi} \omega}+\sqrt{\frac{\pi}{2}} \delta(\omega)\right) \otimes 2 \operatorname{Re}\left[P_{\mathrm{R}}(\omega)\right] \\
=\frac{-i}{\pi} \int_{-\infty}^{\infty} \mathrm{d} \omega_{0} \frac{\operatorname{Re}\left[P_{\mathrm{R}}\left(\omega_{0}\right)\right]}{\omega-\omega_{0}}+\operatorname{Re}\left[P_{\mathrm{R}}(\omega)\right]
\end{aligned}
$$

The real and imaginary parts of the retrieved signal become

$$
\operatorname{Re}\left[\mathrm{FT}^{-1}\left[\theta(t) \mathrm{FT}\left[P_{\mathrm{R}}(\omega)+P_{\mathrm{R}}(\omega)^{*}\right]\right]\right]=\operatorname{Re}\left[P_{\mathrm{R}}(\omega)\right]
$$

$$
\begin{aligned}
\operatorname{Im}\left[\mathrm{FT}^{-1}[\theta(t)\right. & \left.\left.\mathrm{FT}\left[P_{\mathrm{R}}(\omega)+P_{\mathrm{R}}(\omega)^{*}\right]\right]\right] \\
& =\frac{-1}{\pi} \int_{-\infty}^{\infty} \mathrm{d} \omega_{0} \frac{\operatorname{Re}\left[P_{\mathrm{R}}\left(\omega_{0}\right)\right]}{\omega-\omega_{0}}=\operatorname{Im}\left[P_{\mathrm{R}}(\omega)\right]
\end{aligned}
$$

In the imaginary part of eq 14 , we have the Kramers-Kronig relation (eq 12) with $P_{\mathrm{R}}(\omega)$ instead of $\chi(\omega)$. This implies that the current FTSI method is equivalent to the Kramers-Kronig relation.

\section{Experimental Section}

CARS signals are generated with a cavity-dumping Ti: Sapphire oscillator (Cascade, KM Lasers). The repetition rate of the laser is set to $2 \mathrm{MHz}$. The oscillator is optimized to have a laser spectrum with an intense band around $770 \mathrm{~nm}$ and a long tail in the longer wavelengths by carefully adjusting the laser cavity. The laser spectrum of an arm chair shape is chosen to increase the intensity of the narrow band probe pulse to maximize the CARS signal (The original laser spectrum is shown in Figure 7a). Figure 6a shows the experimental setup. The laser pulse is controlled by an all reflective $4 f$ pulse shaper using a 640 pixel dual bank liquid crystal spatial light modulator (SLM-640-D-VN, CRI). The pulse shaper is configured to control both the phase and amplitude of the individual frequency pixels at the Fourier plane. Our pulse shaper is designed to cover a $250 \mathrm{~nm}$ bandwidth centered at $800 \mathrm{~nm}$. The spectral resolution of the pulse shaper, which is limited by the spectral bandwidth of one SLM pixel, is $0.4 \mathrm{~nm}$, corresponding to $5.3 \mathrm{~cm}^{-1}$ at 800 $\mathrm{nm}$. The wavelengths of the laser shorter than $750 \mathrm{~nm}$ are blocked by a razor blade placed just before the SLM. The shaped pulse is focused into the sample, and the generated signal is collected, using two high N.A. water immersion IR objectives (1.2 and 1.0 N.A., respectively, Olympus). The collected signal is filtered by a sharp edge short wave pass filter (740AESP, Omega Optical), coupled into an imaging spectrometer (Holospec $f / 1.8$, Kaiser Optical Systems) and measured with a frame transfer charge-coupled device (CCD) (DV887, Andor). Note that this CCD camera can measure the full vertical binned spectra with an exposure time as short as $20 \mu$ s without a mechanical shutter thanks to the frame transfer CCD architecture.

Spectroscopy experiments are performed with a small drop of the sample sandwiched between two microscope coverslips. The intensity and phase spectrum of our shaped CARS excitation laser pulse is shown in Figure 6b. First, a reference spectrum is measured by applying a transform limited pulse without the probe pulse in Figure 6b. The generated signal with the CARS laser pulse is then normalized with the reference spectrum, Fourier transformed, applied to the Heaviside step function, and inverse Fourier transformed to generate the vibrational spectra (eqs 8 and 11). Vibrational hyperspectral imaging is performed by moving both the laser beam in one direction ( $y$-axis) and translating the sample in the other direction ( $x$-axis) by a piezodriven scanning mirror (SM in Figure 6a, S-334.2SL, PI) and an XY piezo-stage (P-542.2SL, PI), respectively. The laser beam is scanned along the $y$-axis, which translates the collected signal parallel to the input slit of the imaging spectrometer. The beam scanning is synchronized with the CCD to ensure that the scanning laser spot position is correlated with the measured CCD spectrum. After acquiring one line of the image, the sample is 
moved in the perpendicular direction ( $x$-axis) by the sample piezo-stage, and the next line of the image is measured by scanning the laser beam along the $y$-axis again. In this way, our maximum scan speed is limited by the CCD data transfer rate, not by the speed of the sample translation stage since the laser beam can be scanned faster than the sample stage. Although our CCD can measure the CARS signal with an exposure time as short as $20 \mu \mathrm{s}$, the data transfer speed of the $\mathrm{CCD}$ is maximum at $\sim 2 \mathrm{~ms} / \mathrm{spectra}$ and limits the current imaging acquisition speed. With a $1 \mathrm{~ms}$ exposure time per pixel, a $100 \times 100$ pixel image can be taken in $43 \mathrm{~s}$, which includes the CCD exposure, data transfer, and FTSI numerical processes.

The spectral phase of the laser pulse is characterized at the microscope sample position with the single beam SPIDER (spectral phase interferometry for direct electric field reconstruction) technique developed recently. ${ }^{34}$ Here only a brief explanation is given, and more information can be found elsewhere. ${ }^{34}$

(a)

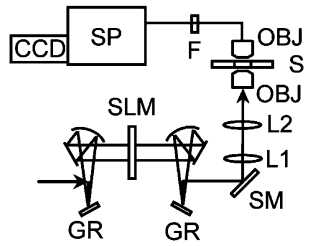

(b)

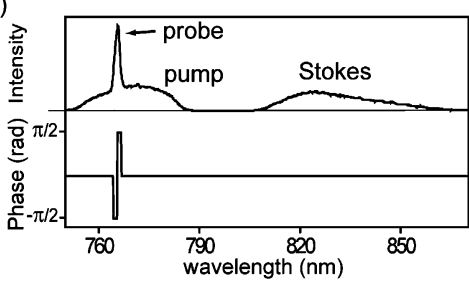

Figure 6. (a) Experimental setup: GR, grating; SLM, dual bank liquid crystal spatial light modulator; SM, beam scanning mirror; L1, scan lens; L2, tube lens; OBJ, microscope objective; S, sample on the XY piezo-stage; F, short wave pass filter; SP, imaging spectrometer. (b) Intensity and phase of the CARS laser pulse used in FTSI-CARS experiments.
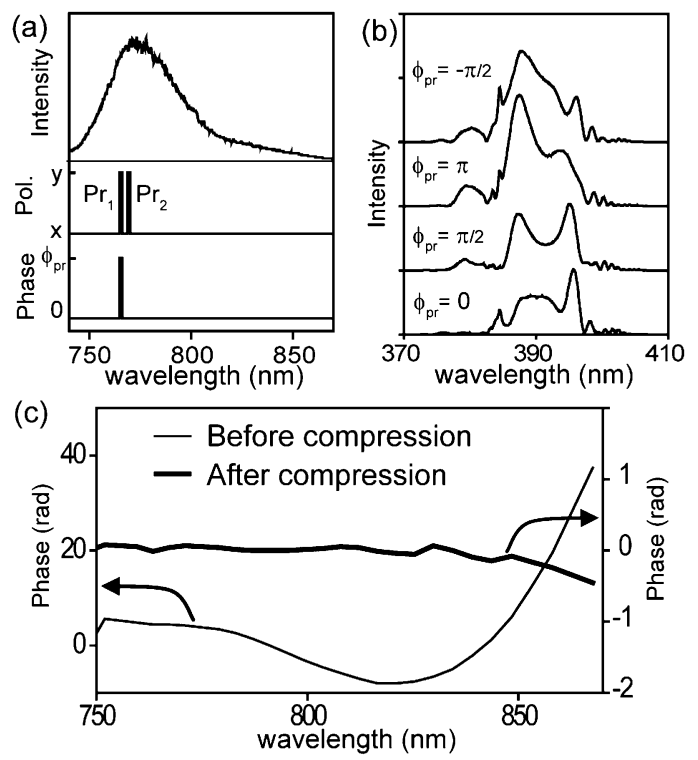

Figure 7. Single-beam SPIDER (a) Spectrum of the original laser pulse (top). Polarization (middle) and phase (bottom) masks used in the pulse characterization. (b) SHG spectra with different phases at $\operatorname{Pr}_{1}\left(\phi_{\mathrm{pr}}=0\right.$, $\pi / 2, \pi$ and $-\pi / 2$ ). The traces are displaced vertically for clarity. (c) The measured spectral phase at the sample position before (thin line) and after (thick line) compressing the laser pulse by applying the compensating phase mask. Note the differences in the vertical scales in panel c.
A phase and polarization mask is applied to select two narrow probe pulses $\left(\operatorname{Pr}_{1}\right.$ and $\operatorname{Pr}_{2}$ in Figure $\left.7 \mathrm{a}\right)$ in the perpendicular polarization direction ( $y$-axis) with respect to the original pulse polarization ( $x$-axis). Intensity, polarization, and phase spectra are shown in Figure 7a. The two $y$-polarized probe pulses and the broad $x$-polarized pulse generate two type-II phase matching second harmonic generation ( $\mathrm{SHG}$ ) spectra with a $50 \mu \mathrm{m}$ thick BBO crystal at the microscope sample position. These two SHG spectra have identical but frequency shifted spectral phases. The detected SHG spectrum is a coherent sum of the two SHG signals, which interfere with each other constructively and destructively along the frequency axis to generate a spectral shear interference pattern as shown in Figure $7 b$. One can obtain the spectral phase of the $x$-polarized broadband laser pulse from the four SHG spectra of different phases at $\operatorname{Pr}_{1}\left(\phi_{\mathrm{pr}}=0, \pi / 2, \pi\right.$ and $-\pi / 2$, where $\phi_{\text {pr }}$ is the phase of $\operatorname{Pr}_{1}$ in Figure 7a) by the following analytical equations:

$$
\begin{aligned}
& \theta(\omega)= \\
& \tan ^{-1}\left[\frac{S\left(\omega+\omega_{\mathrm{p}}, \phi_{\mathrm{pr}}=\pi / 2\right)-S\left(\omega+\omega_{\mathrm{p}}, \phi_{\mathrm{pr}}=-\pi / 2\right)}{S\left(\omega+\omega_{\mathrm{p}}, \phi_{\mathrm{pr}}=0\right)-S\left(\omega+\omega_{\mathrm{p}}, \phi_{\mathrm{pr}}=\pi\right)}\right] \\
& \phi\left(\omega_{0}+n \delta \omega\right)= \\
& \phi\left(\omega_{0}\right)+\sum_{k=1}^{n} \theta\left(\omega_{0}+k \delta \omega\right), \text { where } n \text { is positive integers }
\end{aligned}
$$

where $S\left(\omega, \phi_{\mathrm{pr}}\right)$ is the measured SHG spectra with $\phi_{\mathrm{pr}} . \omega_{\mathrm{p}}$ is the frequency of $\operatorname{Pr}_{1} . \phi(\omega)$ is the spectral phase of the laser pulse at the sample position. $\delta \omega$ is the frequency difference between $\operatorname{Pr}_{1}$ and $\operatorname{Pr}_{2}$. $\omega_{0}$ is set to be $750 \mathrm{~nm}$.

The SHG spectra with four different $\phi_{\mathrm{pr}}$ values are shown in Figure $7 \mathrm{~b}$. Figure $7 \mathrm{c}$ shows the retrieved spectral phase before and after applying the compensating phase mask in the pulse shaper. After applying the compensating phase mask for the measured spectral phase, we are able to compress the laser pulse within 0.3 rad over the entire laser spectral range (from 750 to $860 \mathrm{~nm}$ ). Note that this single beam SPIDER needs a phase and polarization control mode of the SLM by removing the exit polarizer. We measure the spectral phase with the exit polarizer placed in front of the entrance polarizer to match the exact dispersion of the FTSI-CARS experiment. Multiple intrapulse interference phase scan (MIIPS) is also used to verify that the compressed laser pulse is transform limited (data not shown). ${ }^{35}$ Note that we partially compress the laser pulse by moving the second grating in the pulse shaper. This generates the unusual spectral shape as shown in Figure 7c (thin line). This is done because we try to avoid a steeper phase mask structure, which can make the transverse field effect of the SLM worse. Thus, the measured spectral shape (thin line in Figure 7c) is from the dispersion of all the refractive optics (primarily due to the 1.2 N.A. microscope objective) and the pulse shaper. Also note that the two measured spectral phases (before and after pulse compression) have very different vertical scales in Figure 7c.

Liquid samples are prepared by sandwiching a small drop of the liquid between two coverslips. The polymer samples are prepared by melting polymer powders on top of glass coverslips. Polystyrene (PS) and polyethylene terephthalate (PET) polymers are purchased from Aldrich and used as received.

\section{Results}

Figure 8a shows the measured raw CARS signals from cyclohexane with the CARS excitation laser pulse $\left(S_{\pi}\right)$ and the reference excitation pulse $\left(S_{0}\right)$. The CCD exposure time of 1 
ms is used. The pump, Stokes, and probe powers are 1.2, 0.8, and $0.3 \mathrm{~mW}$, respectively, which are measured before the focusing objective. Figure $8 \mathrm{~b}$ shows the normalized CARS signal, which corresponds to the cross term in eq 8 . The FT of this normalized spectrum is shown in Figure 8c (top trace). Applying the Heaviside step function (bottom trace of Figure 8c) and inverse FT yields a homodyne-amplified resonant CARS spectrum with the full complex quantity shown in Figure 8d. The sum of the imaginary part and amplitude of the obtained complex CARS signal shows a Raman-like vibrational spectrum (top trace in Figure 8d). The imaginary part (bottom) and amplitude (middle) of the CARS signals are also shown in Figure 8d.

Figure 9 shows the FTSI-CARS spectra obtained from cyclohexane and toluene with the CCD exposure time of 20 $\mu \mathrm{s}$. Note that this CARS spectrum is taken with only 40 laser pulses since the repetition rate of our laser is $2 \mathrm{MHz}$. The signalto-noise ratio of the CARS spectra in Figure 9a are $\sim 130$ and $\sim 280$ for cyclohexane and toluene, respectively. The same power level is used as the experiment of Figure 8. Note that almost all the previous multiplex CARS experiments have been performed with a more than tens of milliseconds time scale. ${ }^{8,15,23}$ Also note that the vibrational peaks up to $1400 \mathrm{~cm}^{-1}$ are clearly visible in Figures 8d and 9a. This extended vibrational window is possible since we compress our pulse at the sample position and there is negligible ACA in our system, which is discussed later. We use a probe pulse with a bandwidth of 4 SLM pixels (corresponding to $21 \mathrm{~cm}^{-1}$ ), and the spectral resolution is $\sim 20$ $\mathrm{cm}^{-1}$ measured by the full width at the half-maximum of the $1001 \mathrm{~cm}^{-1}$ peak of toluene. One can see that the two neighboring peaks at 1001 and $1028 \mathrm{~cm}^{-1}$ are clearly resolved in our experiment. This result matches with the simulation in Figure $4 \mathrm{~b}$ very well. Figure $9 \mathrm{~b}$ shows the FTSI-CARS spectra of PS and PET film with the CCD exposure time of $100 \mu$ s. Since the polymers have lower photodamage thresholds, we lower the pump power from 1.2 to $0.5 \mathrm{~mW}$ while keeping the same Stokes and probe powers as used in the liquid experiment. The CARS signals of polymers in Figure $9 b$ are noisier than the liquid signals in Figure 9a as a result of the lower pump power, but all the major vibrational peaks of the polymers are clearly visible.

Figure 10a,b shows chemically selective microscope images of a PS/PET mixture film. Note that we take the entire
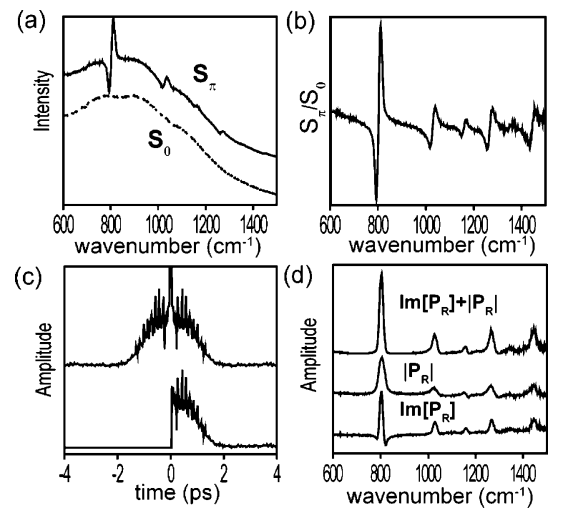

Figure 8. FTSI-CARS experiment: (a) Measured CARS signals from cyclohexane by the CARS ( $S_{\pi}$, top) and the reference $\left(S_{0}\right.$, bottom) laser pulses. (b) Normalized CARS signal $\left(S_{\pi} / S_{0}\right)$. (c) (top) FT of the normalized signal $\left(S_{\pi} / S_{0}\right)$ and (bottom) the zero-filled time profile before the time zero. (d) Imaginary part, amplitude, and a sum of the imaginary part and amplitude for the inverse FT of the zero-filled time profile (the bottom trace in panel c). The traces in panels a, c, and $\mathrm{d}$ are vertically displaced for clarity. The CCD exposure time is $1 \mathrm{~ms}$. vibrational spectra at every spatial point. We construct the images by integrating 970 to $1040 \mathrm{~cm}^{-1}$ for PS and 1060 to $1140 \mathrm{~cm}^{-1}$ for PET in the 10000 vibrational spectra. The pixel size in these images is $300 \mathrm{~nm}$. An exposure time of $1 \mathrm{~ms}$ per pixel and the same laser power as Figure $9 \mathrm{~b}$ are used here. The total image acquisition time is $43 \mathrm{~s}$, as described in the Experimental Section. Note that the actual CCD exposure times for 10000 spectra is $20 \mathrm{~s}$, but the data transferring and numerical process takes an additional $23 \mathrm{~s}$. One can clearly see that circular PS domains are surrounded by a PET domain in Figure 10.

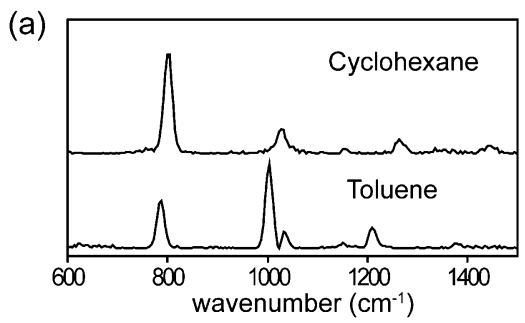

(b)

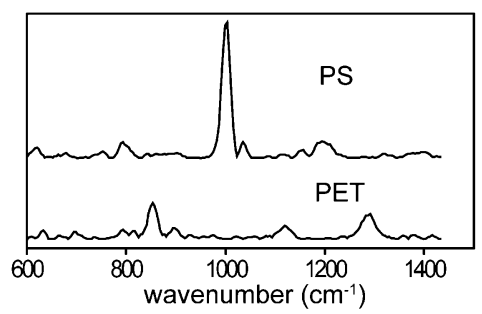

Figure 9. (a) FTSI-CARS spectra of cyclohexane and toluene taken under the microscope setup with $20 \mu$ s CCD exposure time. (b) FTSICARS spectra of PS and PET with $100 \mu$ s CCD exposure time. Each trace is displaced vertically for clarity. (a) $1000 \mathrm{~cm}^{-1}$

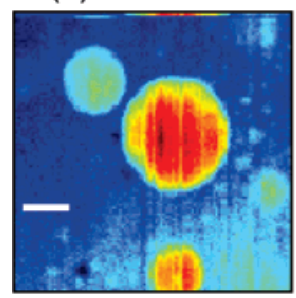

(b) $1100 \mathrm{~cm}^{-1}$

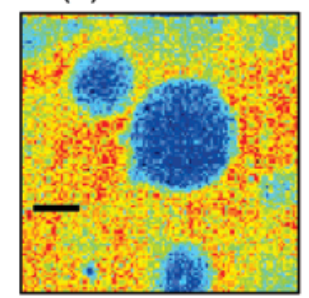

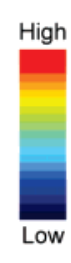

Figure 10. Chemical imaging of PS and PET mixture film. Images are constructed with peaks at (a) $1000 \mathrm{~cm}^{-1}$ (PS) and (b) $1100 \mathrm{~cm}^{-1}$ (PET), respectively. The scale bar is $5 \mu \mathrm{m}$. The images have $100 \times$ 100 pixels, and the pixel size is $300 \mathrm{~nm} \times 300 \mathrm{~nm}$. Note that these images are constructed from a single experimental scan, where each pixel in the image contains the complete spectrum over $500-1400 \mathrm{~cm}^{-1}$. The total image acquisition time is $43 \mathrm{~s}$.

\section{Discussion}

To optimize multiplex CARS signal generation, one needs to consider several technical issues such as pulse compression, ACA and optimal power distribution among the pump, and Stokes and probe pulses. The first requirement for the maximum multiplex CARS signal is that the pump, Stokes, and probe pulses should have a good temporal and spatial overlap. Since we are using short and long wavelength parts of a single broadband laser spectrum, we need to compensate the spectral phase not only of the linear chirp ( $\omega^{2}$ term in the spectral phase) but also all the high-order terms. In this experiment, we compress the laser pulse by the single beam SPIDER as described earlier. ${ }^{34}$ It obtains the spectral phase analytically without the polynomial expansion of the spectral phase. This method greatly improves the sensitivity of the CARS signal over the $1100-1400 \mathrm{~cm}^{-1}$ range. Also, ACA leads the focuses of 
(a)

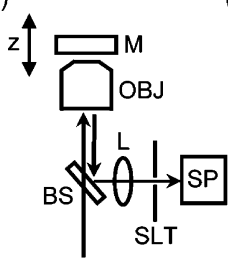

(b)

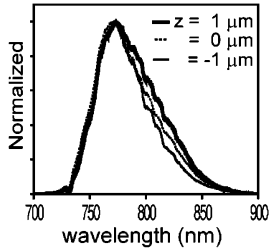

Figure 11. (a) Experimental setup for ACA characterization: BS, 50: 50 beam splitter; L, tube lens (focal length $=20 \mathrm{~cm}$ ); SLT, $20 \mu \mathrm{m}$ slit (inside the spectrometer); SP, spectrometer; OBJ, 1.2 N.A. microscope objective; $\mathrm{M}$, mirror on top of a sample stage with a motorized vertical actuator. (b) Normalized laser spectra obtained with three different vertical mirror positions at $z=-1,0$, and $1 \mu \mathrm{m} . z=0$ is the focus of the objective lens.

the pump and Stokes pulses, which are displaced at different vertical positions, leading to a poor spatial overlap between the two excitation laser pulses. Another issue is that the ultimate detection sensitivity is limited by the maximum laser power that a sample can tolerate without photodamage. Since we are using a very short pulse, the main photodamage mechanism is nonlinear absorption. So a clever choice of the power distribution among the three pulses (pump, Stokes, and probe) should be made.

One of the general problems in nonlinear microscopy with a broadband laser pulse is ACA. It prevents the spatial overlap between different frequency components to reduce the signal strength. We characterize the ACA at the focus of our microscope by an epidetected confocal microspectroscopy of a reflected laser pulse. Figure 11a shows the experimental setup. We place a mirror at the sample position and move it vertically by a computer-controlled motorized actuator (Z606, Thorlabs). The reflected laser beam is collimated by the same objective in the epi-direction, split by a 50:50 beam splitter and focused into a spectrometer with a $20 \mu \mathrm{m}$ slit (USB4000, Ocean Optics). ACA will cause different spectral shapes of the measured laser spectra along the vertical mirror position $(z)$ in this setup since the different frequency components will focus at the different vertical positions if there is a significant ACA. Figure 11b shows the normalized spectra at three different vertical mirror positions $(z=-1,0$, and $1 \mu \mathrm{m} . z=0$ is the focus of the objective). One can see that there is no significant ACA in the current experimental setup. It is worth noting that we use near-infrared optimized achromat lenses (Thorlabs) in our entire experimental setup and an apochromatic microscope objective (UPLSAPO60XW, Olympus), which is designed to be free of ACA over the wavelength region of the laser used here.

With a short laser pulse with a 20 fs pulse duration (the pulse duration of the original laser spectrum shown in Figure 7a), the power density at the focus of 1.2 NA objective can reach 5 $\times 10^{12} \mathrm{~W} / \mathrm{cm}^{2}$ with $1 \mathrm{~mW}$ of laser power at a $2 \mathrm{MHz}$ repetition rate. This is a high enough power density to cause multiphoton absorption, continuum generation, and even optical breakdown. So the maximum sensitivity of our CARS microscopy is limited by the photodamage of the sample. ${ }^{36}$ Removing the laser frequency components from 780 to $810 \mathrm{~nm}$ (as shown in Figure 6b) makes the pulse duration significantly longer while keeping the same CARS sensitivity over the vibrational window from 600 to $1400 \mathrm{~cm}^{-1}$. We also control the powers of each three pulses independently by the amplitude pulse shaping. With a fixed total laser power, the optimal power distribution is such that all three pulses have equal powers. ${ }^{1}$ This means that the optimal amplitude shape is close to the one shown in Figure 5a with equal powers over the broad pump and Stokes pulses and the narrow probe pulse. Among the three CARS excitation laser

pulses, however, the probe pulse has a much longer pulse duration $(\sim \mathrm{ps})$, and its contribution to the photodamage is minimal compared to that of the other two short pulses (pump and Stokes). So we use the maximum available power at the probe pulse as shown in Figure $6 \mathrm{~b}$. We also find that the photodamage is more severe with the pump laser pulse. This is due to the short wavelength of the pump beam and has been reported previously. ${ }^{29}$ Therefore, we primarily control the bandwidth and amplitude of the pump pulse while monitoring the CARS signals and photodamage. One can reduce the photodamage effect more by applying controlled linear chirp to both the pump and Stokes pulses. We found that our FTSICARS method works reasonably well with applied linear chirps up to $500 \mathrm{fs}^{2}$ of the pump and Stokes pulse separately (data not shown). Note that the pump and Stokes pulses still need to have a good time overlap.

It is worth comparing the nonlinearity of power dependence for the CARS and sample photodamage. There are several reports for nonlinear photodamage studied by cell differentiation, ${ }^{37}$ changes in $\mathrm{Ca}^{2+}$ level, and the degranulation reaction. ${ }^{36}$ Photodamage nonlinearity is reported from $2.5^{36}$ to $1.1,{ }^{29}$ which is lower than 3, the nonlinearity of CARS. If the nonlinearity of sample photodamage is lower than that of CARS, a laser with a lower repetition rate and higher peak power is preferred in the CARS experiment since it can generate more signals than the laser with a higher repetition rate and lower peak power. ${ }^{4}$ We compare the CARS signals with two different outputs of our laser: the cavity-dumping output $(2 \mathrm{MHz})$ and the modelocking output $(90 \mathrm{MHz}$ ) (data not shown). This is possible since our laser can also be operated as a normal Ti:Sapphire oscillator laser. We find that the laser pulse with a lower repetition rate and higher peak power (cavity-dumping output) has a better CARS sensitivity by comparing the CARS signals with the maximum allowable laser power determined by the photodamage of polymers.

Our technique is essentially a common path spectral interferometry. The local oscillator (the non-resonant signal) and the CARS signal are generated by the same laser pulse and never separated from each other. This greatly helps the stability of the experiment. It is known that typical interferometric experiments are sensitive to air flows or environmental vibrations. We find that air fluctuation inside the pulse shaper indeed affects our FTSI-CARS seriously. Enclosing the pulse shaper is necessary to eliminate the instability of the experiment. After the pulse shaper, however, the experiment is very stable and insensitive to ambient air currents or small vibrations.

\section{Conclusion}

In this paper, we report a significant sensitivity improvement of the multiplex CARS technique by optimizing both the amplitude and phase shape of the laser pulse. Controlling the power distribution of the pump, Stokes and probe pulses to optimize the CARS signal generation enable us to measure the vibrational spectrum of liquid and polymer samples within 20$100 \mu \mathrm{s}$. The FTSI-CARS method extracts the full complex quantity of the complex CARS vibrational spectrum to achieve a spectral resolution of $20 \mathrm{~cm}^{-1}$ with a spontaneous Ramanlike spectral line shape. The technique requires a slightly involved data analysis process but has a simple and very stable experimental setup. It should be useful for probing a local chemical identity in inhomogeneous samples.

Acknowledgment. S.H.L. thanks Oh Kyu Yoon for the discussion of the relationship between the FTSI and the 
Kramers-Kronig relations. The authors gratefully acknowledge the Welch Foundation for support of personnel. The instrumentation used in this research is supported by the start-up fund, Department of Chemistry and Biochemistry, University of Texas at Austin.

\section{References and Notes}

(1) Cheng, J. X.; Xie, X. S. J. Phys. Chem. B 2004, 108, 827.

(2) Levenson, M. D.; Kano, S. S. Introduction to Nonlinear Spectroscopy; Academic Press: San Diego, CA, 1988.

(3) Potma, E. O.; Xie, X. S.; Muntean, L.; Preusser, J.; Jones, D.; Ye, J.; Leone, S. R.; Hinsberg, W. D.; Schade, W. J. Phys. Chem. B 2004, 108, 1296.

(4) Cheng, J. X. Appl. Spectrosc. 2007, 61, 197A

(5) Cui, M.; Joffre, L.; Skodack, J.; Ogilvie, J. P. Opt. Exp. 2006, 14, 8448 .

(6) Evans, C. L.; Potma, E. O.; Xie, X. S. Opt. Lett. 2004, 29, 2923.

(7) Ganikhanov, F.; Evans, C.; Saar, B. G.; Xie, X. S. Opt. Lett. 2006, $31,1872$.

(8) Kee, T. W.; Cicerone, M. T. Opt. Lett. 2004, 29, 2701.

(9) Knutsen, K. P.; Messer, B. M.; Onorato, R. M.; Saykally, R. J. J. Phys. Chem. B 2006, 110, 5854 .

(10) Lim, S.-H.; Caster, A.; Leone, S. R. Opt. Lett. 2007, 32, 1332.

(11) Marks, D. L.; Boppart, S. A. Phys. Rev. Lett. 2004, 92, 123905.

(12) Oron, D.; Dudovich, N.; Silberberg, Y. Phys. Rev. Lett. 2003, 90 , 213902.

(13) Oron, D.; Dudovich, N.; Silberberg, Y. Phys. Rev. Lett. 2002, 89, 273001.

(14) Potma, E. O.; Evans, C. L.; Xie, X. S. Opt. Lett. 2006, 31, 241.

(15) Muller, M.; Schins, J. M. J. Phys. Chem. B 2002, 106, 3715.

(16) Zumbusch, A.; Holtom, G. R.; Xie, X. S. Phys. Rev. Lett. 1999, 82,4142 .

(17) Cheng, J. X.; Volkmer, A.; Book, L. D.; Xie, X. S. J. Phys. Chem. B 2001, 105, 1277.
(18) Li, L.; Wang, H.; Chen, J. X. Biophys. J. 2005, 89, 3480.

(19) Evans, C. L.; Potma, E. O.; Puoris'hagg, M.; Cote, D.; Lin, C. P.; Xie, X. S. Proc. Natl. Acad. Sci. U.S.A. 2005, 102, 16807.

(20) Nan, X. L.; Potma, E. O.; Xie, X. S. Biophys. J. 2006, 91, 728

(21) Cheng, J. X.; Volkmer, A.; Book, L. D.; Xie, X. S. J. Phys. Chem. B 2002, 106, 8493 .

(22) Lim, S.-H.; Caster, A. G.; Leone, S. R. Phys. Rev. A 2005, 72, 041803.

(23) Lim, S.-H.; Caster, A. G.; Leone, S. R. J. Phys. Chem. B 2006 110,5196

(24) Wurpel, G. W. H.; Schins, J. M.; Muller, M. J. Phys. Chem. B 2004, 108, 3400 .

(25) Pestov, D.; Murawski, R. K.; Ariunbold, G. O.; Wang, X.; Zhi, M.; Sokolov, A. V.; Sautenkov, V. A.; Rostovtsev, Y. V.; Dogariu, A.; Huang, Y.; Scully, M. O. Science 2007, 316, 265.

(26) Wurpel, G. W. H.; Muller, M. Chem. Phys. Lett. 2006, 425, 336.

(27) Marks, D. L.; Vinegoni, C.; Bredfeldt, J. S.; Boppart, S. A. Appl. Phys. Lett. 2004, 85, 5787.

(28) Dudovich, N.; Oron, D.; Silberberg, Y. J. Chem. Phys. 2003, 118, 9208.

(29) Fu, Y.; Wang, H.; Shi, R.; Chen, J. X. Opt. Exp. 2006, 14, 3942.

(30) Oron, D.; Dudovich, N.; Yelin, D.; Silberberg, Y. Phys. Rev. Lett. 2002, 88,63004 .

(31) Lin-Vien, D.; Colthup, N. B.; Fateley, W. G.; Grasselli, J. G. The Handbook of Infrared and Raman Characteristic Frequencies of Organic Molecules; Academic Press: San Diego, CA, 1991.

(32) Arfken, G. B. Mathematical Methods for Physicists, 5th ed.; Academic Press: San Diego, CA, 2001.

(33) Lepetit, L.; Cheriaux, G.; Joffre, M. J. Opt. Soc. Am. B 1995, 12, 2467.

(34) Chen, B.-C.; Lim, S.-H. Opt. Lett. 2007, 32, 2411.

(35) Xu, B.; Gunn, J. M.; Dela Cruz, J. M.; Lozovoy, V. V.; Dantus, M. J. Opt. Soc. Am. B 2006, 23, 750.

(36) Hopt, A.; Neher, E. Biophys. J. 2001, 80, 2029.

(37) Konig, K. J. Microsc. 2000, 200, 83. 